\title{
Successful percutaneous coronary intervention of a thrombosed Cabrol graft after intraoperative cardiac arrest
}

\author{
Motaz Moussa MD, Rahul Bhindi MD, Amir Ravandi MD PhD
}

\begin{abstract}
M Moussa, R Bhindi, A Ravandi. Successful percutaneous coronary intervention of a thrombosed Cabrol graft after intraoperative cardiac arrest. Curr Res Cardiol 2014;1(2):111-113.
\end{abstract}

The Cabrol technique is used to reimplant coronary arteries after aortic root replacement using a Dacron graft interposed between the aortic root graft and the native coronary artery. Although stenosis of the coronary graft anastomosis has been described, the authors present a case involving acute graft closure due to kinking of the Cabrol graft treated with percutaneous coronary intervention and thrombectomy.

A 68-year-old man with a history of Bentall repair was admitted with graft and aortic valve endocarditis. He underwent a repeat aortic root reconstruction using the Cabrol procedure for the reanastomosis of the right coronary and left main coronary artery (LMCA) to the aortic graft. He returned to the operating room $48 \mathrm{~h}$ later for repair of a leaking subannular pericardial patch. During this procedure, he experienced a cardiac arrest and Doppler ultrasonography demonstrated poor flow to the left coronary interposition graft.

The Cabrol technique is used to reimplant coronary arteries after 1 aortic root replacement using a Dacron graft interposed between the aortic root graft and the native coronary artery. Although stenosis of the coronary graft anastomosis has been described previously, we present a case of acute graft closure due to kinking of the Dacron graft that was successfully treated with percutaneous coronary intervention (PCI) and thrombectomy.

\section{CASE PRESENTATION}

A 68-year-old man with a history of Bentall repair was admitted to hospital with graft and aortic valve endocarditis. He underwent a repeat aortic root reconstruction using the Cabrol procedure for the reanastomosis of the right coronary and left main coronary artery to the aortic graft. He returned to the operating room $48 \mathrm{~h}$ later for repair of a leaking subannular pericardial patch. During this procedure, he experienced a cardiac arrest and Doppler ultrasound demonstrated poor flow to the left coronary interposition graft. Soon thereafter, the patient was placed on extracorporeal membrane oxygenation and sent to the cardiac catheterization laboratory on an urgent basis for coronary angiography. Coronary angiography revealed a thrombosed Cabrol graft to the left main coronary artery. After engaging the graft with a 7 Fr multipurpose guiding catheter, multiple rounds of thrombectomy were performed using the Pronto thrombectomy catheter, re-establishing Thrombosis in Myocardial Infarction (TIMI) grade 3 flow. Post-thrombectomy, a kink was revealed at the proximal end of the Dacron graft, which was likely a result of mechanical manipulation during surgery (Figure 1). Given the hemodynamic instability of the patient, the kink was treated within the Dacron graft with PCI as opposed to surgical repair (Figure 2). The kinked segment was stented with a $6.0 \mathrm{~mm} \times 29 \mathrm{~mm}$ Omnilink baremetal stent (BMS) (Abbott Vascular, USA) and a $5.0 \mathrm{~mm} \times 20 \mathrm{~mm}$ biokinetic BMS in an overlapping manner (Figure 3 ) and postdilated
The patient was placed on extracorporeal membrane oxygenation and sent for urgent coronary angiography. Coronary angiography revealed a thrombosed Cabrol graft to the LMCA. Multiple rounds of thombectomy using the Pronto thrombectomy catheter re-established Thrombosis in Myocardial Infarction grade 3 flow and revealed a kink at the proximal end of the graft. Given the hemodynamic instability of the patient, a decision was made to treat the kink within the graft with percutaneous coronary intervention (PCI). The kinked segment was stented with $6.0 \mathrm{~mm} \times 29 \mathrm{~mm}$ and $5.0 \mathrm{~mm}$ $\times 20 \mathrm{~mm}$ bare-metal stents in an overlapping fashion. This resulted in Thrombosis in Myocardial Infarction grade 3 flow within the graft and the entire left coronary system with improved hemodynamics. The present case is, to the authors' knowledge, the first to document a LMCA graft occlusion intraoperatively, resulting in cardiac arrest with successful reperfusion established by PCI. In conclusion, PCI is an effective technique to treat an acute occlusion of a Cabrol graft in a hemodynamically unstable patient.

\section{Key Words: Angioplasty; Cabrol graft; Thrombectomy}

with a $7.0 \mathrm{~mm}$ balloon. This resulted in TIMI 3 flow within the Dacron graft and the entire left coronary system, with improved hemodynamics (Figure 4). Given that the etiology of the kink was likely mechanical in nature, the patient was placed on the standard anticoagulation regimen for a BMS including acetylsalicylic acid and clopidogrel. Unfortunately, after transfer to the intensive care unit, the patient developed severe biventricular dysfunction and complete thrombosis of the aortic valve. The decision was made to palliate and the patient passed away within a few days. To the authors' knowledge, the present case is the first to document an intraoperative Dacron graft occlusion (post-Cabrol procedure)

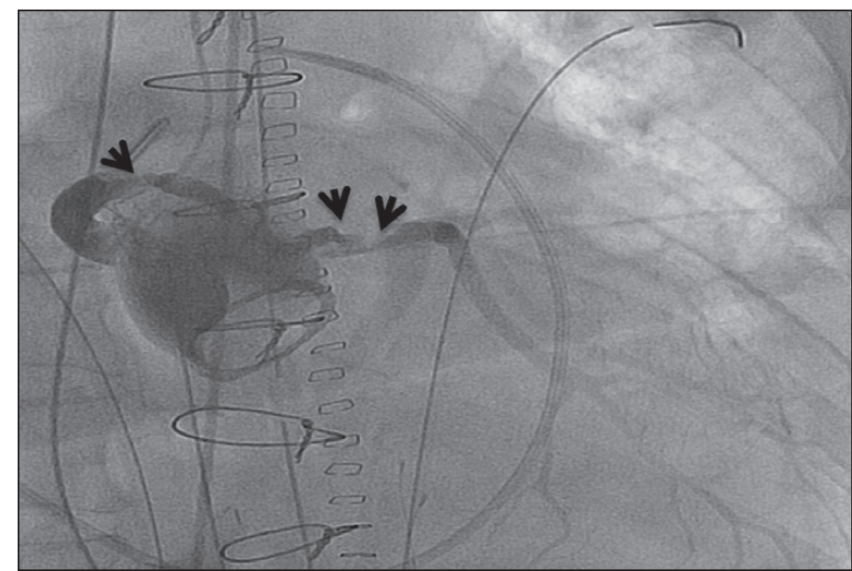

Figure 1) Coronary angiography of thrombosed Cabrol graft. The graft to the left main coronary artery was engaged with a $7 \mathrm{Fr}$ multipurpose guiding catheter. Diffuse thrombosis is evident in the left Dacron graft (black arrows)

Division of Cardiology, St Boniface Hospital, University of Manitoba Winnipeg, Manitoba

Correspondence: Dr Amir Ravandi, Room Y3508, Bergen Cardiac Care Centre, St Boniface General Hospital, 409 Tache Avenue, Winnipeg, Manitoba R2H 2A6. Telephone 204-237-2315, fax 204-237-2023, e-mail aravandi@sbgh.mb.ca 


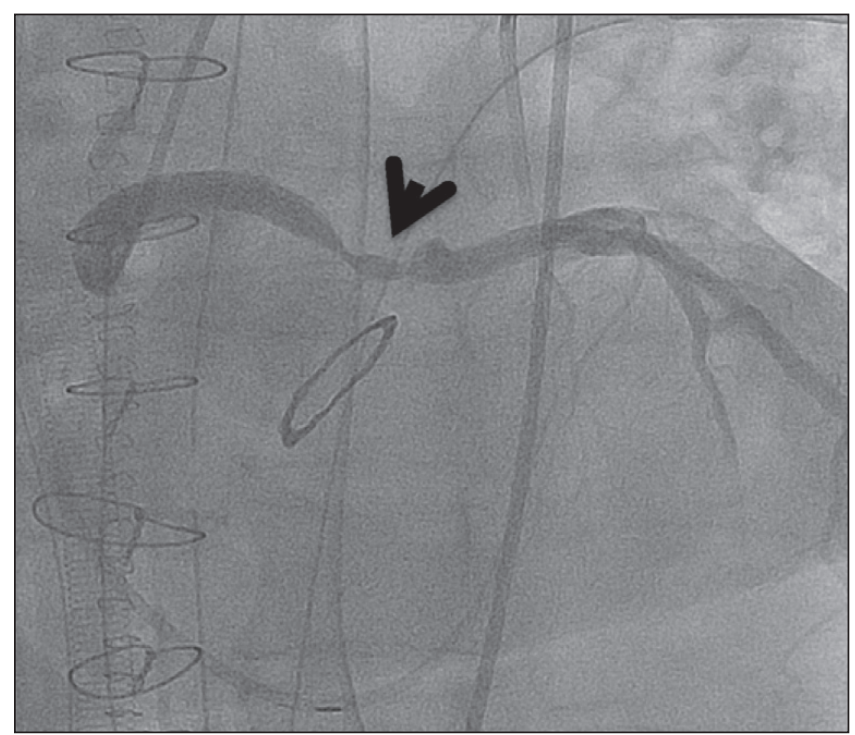

Figure 2) Multiple rounds of thombectomy using the Pronto thrombectomy catheter (Vascular Solutions, USA) reestablished Thrombosis in Myocardial Infarction 3 flow. It also revealed a possible kink or mechanical defect in the proximal end of the graft (black arrow)

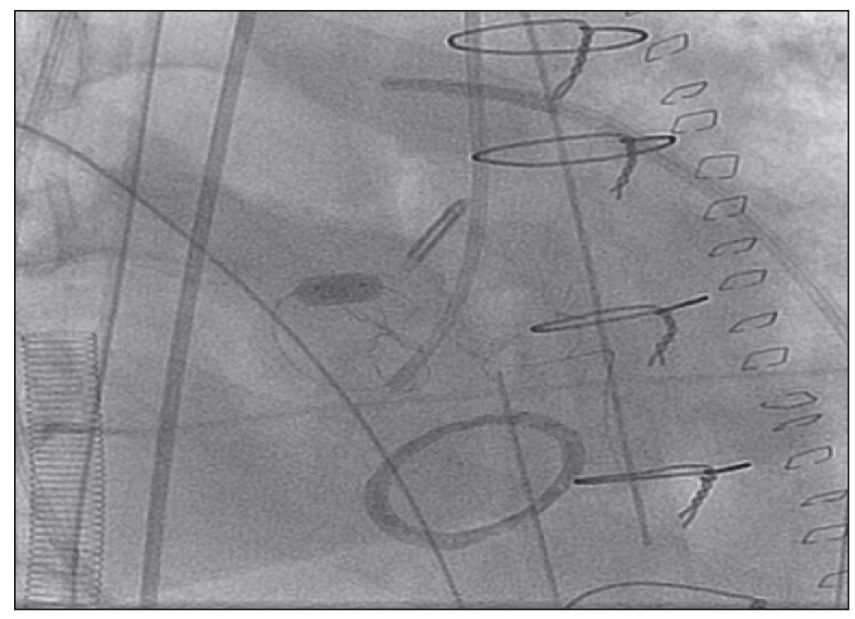

Figure 3) A $6.0 \mathrm{~mm} \times 29 \mathrm{~mm}$ Omnilink lead bare-metal stent (Abbott Vascular, USA) was deployed in the proximal end of the graft. This shifted the kink distally, requiring an additional prokinetic $5.0 \mathrm{~mm} \times 20 \mathrm{~mm}$ baremetal stent

resulting in cardiac arrest with successful reperfusion established by PCI. In conclusion, PCI is an effective technique to treat an acute occlusion of a Cabrol graft in a hemodynamically unstable patient.

\section{DISCUSSION}

Current surgical therapies for patients with aortic root dissections or aneurysms are complex and fastidious in nature. The Cabrol procedure is one such surgical procedure to repair a dissecting aortic aneurysm $(1,2)$. It is rarely used as a first-line surgical technique to reimplant coronary ostia in ascending aorta reconstruction (3). The Cabrol technique is often required when there is an anatomically fixed coronary ostia and scarring from previous operations, button calcifications, coronary aneurysms and/or coronary anomalies. The technique uses a Dacron or Gore-Tex graft to connect the coronary arteries to the aortic root (4). The graft is attached to the aortic root using an end-to-side technique and to the coronary ostia using an end-to-end technique (5). The mortality rate has been reported to be

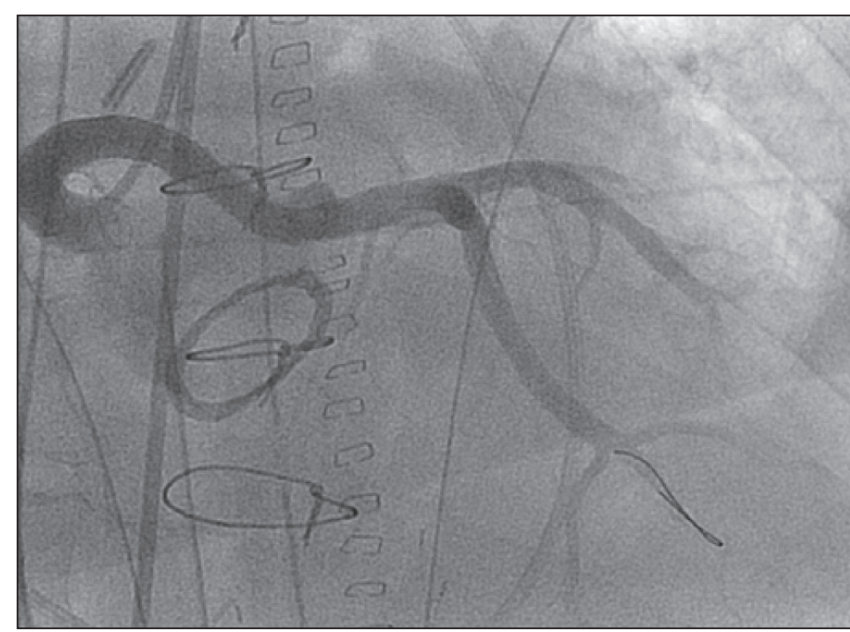

Figure 4) Image demonstrating brisk flow within the graft postintervention

as high as $20 \%$, which is significantly higher than other aortic repair techniques (6). This may be due to the meticulous nature of the Cabrol procedure and the choice of method for the mobilization and reimplantation of the coronary artery ostia during complex composite graft replacement of the aorta.

Due to stenosis and thrombosis with resultant occlusion of the interposed graft and methodological limitations with the Cabrol technique, the Bentall procedure for coronary artery reimplantation remains the gold standard. Based on several studies, 30-day mortality rates from a Cabrol procedure ranged from $4 \%$ to $11 \%$, compared with $1.9 \%$ with the Bentall procedure $(7,8)$.

Although there are reports examining short- and long-term outcome data for the Cabrol technique, these studies are often hard to interpret due to a myriad of issues: the variability of underlying pathology, inconsistencies in reporting nonfatal complications, and patient selection bias toward more complex pathology and physiology (3). As a result, rates of interposition Dacron graft stenosis due to diverse pathology varies and has been reported to be as high as $44 \%$ and as low as $2 \%$, although no kinking has been reported $(9,10)$. As with our patient, the risk for complication and mortality with repeat surgical intervention was deemed to be considerably higher when compared with PCI, making PCI the procedure of choice. As with angioplasty in all fresh surgical grafts, avoidance of the anastomotic site due to potential dehiscence must be considered.

The pathophysiological mechanism of thrombus formation in this patient remains unclear; however, our case suggests that thrombus formation was likely secondary to the kink at the proximal end of the graft, with resultant sluggish flow caused by operative cardiac manipulation. Given the posterior position and its longer length, the left Cabrol graft is likely to be more prone to kink formation during intraoperative manipulation of the heart. Another potential explanation is inadequate anticoagulation status post-cardiac surgery. Unfortunately, there are no guidelines or available evidence with regard to optimal anticoagulation therapy after a Cabrol procedure.

As such, caution needs to be exercised when manipulating the heart after an aortic root repair. The present case once again illustrates the necessity for interventional cardiologists to understand the unique anatomy in this population and the need to maintain adequate patency in Dacron grafts following a Cabrol procedure.

\section{REFERENCES}

1. Hussain F, Ducas J, Gosal T. Emergent percutaneous intervention with a drug-eluting stent of a Cabrol graft-to-left main anastomosis during a non-ST-elevation infarction in a patient with Marfan's syndrome. J Invasive Cardiol 2006;18:E250-2.

2. Jang WI, Jeong JO, Ahn KT, et al. A successful primary percutaneous coronary intervention twelve days after a Cabrol composite graft operation in Marfan syndrome. Korean Circ J 2010;40:247-50. 
3. Kourliouros A, Soni M, Rasoli S, et al. Evolution and current applications of the Cabrol procedure and its modifications. Ann Thorac Surg 2011;91:1636-41.

4. Ziganshin BA, Williams FE, Tranquilli M, Elefteriades JA. Midterm experience with modified Cabrol procedure: Safe and durable for complex aortic root replacement. J Thorac Cardiovasc Surg 2014;147:1233-9.

5. Midulla PS, Ergin A, Galla J, et al. Three faces of the Bentall procedure. J Card Surg 1994;9:466-81.

6. Bachet J, Termignon JL, Goudot B, et al. Aortic root replacement with a composite graft. Factors influencing immediate and long-term results. Eur J Cardiothorac Surg 1996;10:207-13.

7. Senanayake EL, Cooper GJ. Indirect re-implantation of the left coronary artery during aortic surgery. J Card Surg 2012;27:205-10.
8. Gelsomino S, Frassani R, Da Col P, et al. A long-term experience with the Cabrol root replacement technique for the management of ascending aortic aneurysms and dissections. Ann Thorac Surg 2003;75:126-31.

9. Mestres CA, Betriu A, Pomar JL. Occlusion of the coronary perfusion graft: An exceptional complication after reconstruction of the ascending aorta with the Cabrol technique. J Thorac Cardiovasc Surg 1989;98:302-3.

10. Guilmet D, Bonnet N, Saal JP, Le Houerou D, Ghorayeb G. [Long term survival with the Bentall button operation in 150 patients]. Arch Mal Coeur Vaiss 2004;97:83-91. 